\title{
Controlling the Gate: The Functions of the Cytoskeleton in Stomatal Movement
}

\author{
Yihao $\mathrm{Li}^{1}$, Xin Zhang ${ }^{1}$, Yi Zhang ${ }^{2 *}$ and Haiyun Ren ${ }^{1,2 *}$ \\ ${ }^{1}$ Center for Biological Science and Technology, Guangdong Zhuhai-Macao Joint Biotech Laboratory, Advanced Institute of \\ Natural Science, Beijing Normal University, Zhuhai, China, ${ }^{2}$ Key Laboratory of Cell Proliferation and Regulation Biology of \\ Ministry of Education, College of Life Sciences, Beijing Normal University, Beijing, China
}

Stomata are specialized epidermal structures composed of two guard cells and are involved in gas and water exchange between plants and the environment and pathogen entry into the plant interior. Stomatal movement is a response to many internal and external stimuli to increase adaptability to environmental change. The cytoskeleton, including actin filaments and microtubules, is highly dynamic in guard cells during stomatal movement, and the destruction of the cytoskeleton interferes with stomatal movement. In this review, we discuss

OPEN ACCESS

Edited by:

Ying Fu,

China Agricultural University, China

Reviewed by:

Takashi Hashimoto,

Nara Institute of Science and

Technology (NAIST), Japan

Yun Xiang,

Lanzhou University, China

*Correspondence:

Yi Zhang

yi.zhang@bnu.edu.cn

Haiyun Ren

hren@bnu.edu.cn

Specialty section:

This article was submitted to

Plant Physiology,

a section of the journal

Frontiers in Plant Science

Received: 06 January 2022

Accepted: 26 January 2022

Published: 23 February 2022

Citation:

Li Y, Zhang $X$, Zhang $Y$ and Ren $H$ (2022) Controlling the Gate: The Functions of the Cytoskeleton in

Stomatal Movement.

Front. Plant Sci. 13:849729.

doi: 10.3389/fp/s.2022.849729 recent progress on the organization and dynamics of actin filaments and microtubule network in guard cells, and we pay special attention to cytoskeletal-associated protein-mediated cytoskeletal rearrangements during stomatal movement. We also discuss the potential mechanisms of stomatal movement in relation to the cytoskeleton and attempt to provide a foundation for further research in this field.

Keywords: stomatal movement, actin filament, microtubule, actin-binding proteins, microtubule-associated proteins

\section{INTRODUCTION}

The plant leaf epidermis and cuticle protect water against transpiration in relatively dry terrestrial environments but also limit gas exchange with the external environment for photosynthesis. Plants have evolved stomata on the leaf and stem epidermis; these structures consist of two kidney-shaped or dumbbell-shaped guard cells and are responsible for $95 \%$ gas exchange between the external atmosphere and the interior of the leaf (Keenan et al., 2013; Lawson and Matthews, 2020). Furthermore, stomata provide major sites for pathogen entry (Gudesblat et al., 2009; Zeng et al., 2010; Melotto et al., 2017). Plants are able to adjust stomatal opening and closure in response to environmental changes (Hetherington and Woodward, 2003; Murata et al., 2015). Hence, an attractive research system for investigations of signal transduction and physiological responses has been developed on the basis of stomatal functions.

The key factor driving stomatal movement is the turgor pressure change provoked by ions and water across plasma and vacuolar membranes, giving rise to swelling or deflation of the cells and opening or closing of the pores (Kollist et al., 2014; Woolfenden et al., 2018; Lawson and Matthews, 2020). In recent decades, a series of ion channels and transporters and their upstream regulators fine-tuning osmotic pressure in guard cells have been identified (Kollist et al., 2014; Lawson and Matthews, 2020). The activities of these channels and transporters depend on voltage sensing, ligand binding, or protein posttranslational modification. For example, the $\mathrm{H}^{+}$-ATPase 
AHA1 is activated by blue light, which leads to hyperpolarization of the plasma membrane in guard cells (Kinoshita and Shimazaki, 1999; Kinoshita et al., 2001; Hayashi et al., 2011). The change in membrane potential drives $\mathrm{K}^{+}$influx through KAT1/2voltagegated $\mathrm{K}^{+}$channels, accompanied by anion $\mathrm{Cl}^{-}$and malate influx (Lebaudy et al., 2010; Yamauchi et al., 2016). Increased levels of osmotically active substances further increase water uptake, resulting in the inflation of guard cells and stomatal opening.

Emerging studies provide evidences indicating that the cytoskeleton, including actin filaments (AFs) and microtubules (MTs), is considered as an important factor involved in stomatal movement, possibly via affecting turgor pressure in guard cells (Zhang and Fan, 2009; Khanna et al., 2014). The cytoskeleton participates in cell division and cell wall synthesis, which affect guard cell shape, structure, and mechanics (Galatis and Apostolakos, 2004; Panteris et al., 2018; Woolfenden et al., 2018; Muroyama et al., 2020). MTs guide cellulose synthesis complexes and determine cellulose microfibril orientation, which might provide high tensile strength in guard cells during stomatal movement (Woolfenden et al., 2018). On the other hand, the cytoskeleton undergoes rapid dynamic changes during stomatal movement, and stomatal movement is inhibited in cytoskeleton-deficient plants. In this review, we discuss current knowledge of cytoskeletal dynamics and their regulation in guard cells and aim to provide novel insights into the mechanisms of cytoskeleton-dependent stomatal movement.

\section{DYNAMICS AND FUNCTIONS OF ACTIN FILAMENTS IN GUARD CELLS}

Pharmacological inhibitors are commonly used to study the dynamics and functions of cytoskeleton and have demonstrated a prerequisite for actin remodeling in stomatal movement. Treatment with the AF stabilizers, such as phalloidin or jasplakinolide, inhibits stomatal closure induced by $\mathrm{ABA}, \mathrm{H}_{2} \mathrm{O}_{2}$, and darkness, and phalloidin (but not jasplakinolide) also inhibited light-induced stomatal opening (Kim et al., 1995; MacRobbie and Kurup, 2007; Li et al., 2014). In contrast, the application of the AF-depolymerizing agent latrunculin B (but not cytochalasin B or D) accelerates ABA-induced stomatal closure, whereas cytochalasin B and D facilitate light-induced stomatal opening (Kim et al., 1995; MacRobbie and Kurup, 2007). The different effects of phalloidin versus jasplakinolide treatment and latrunculin $B$ versus cytochalasin treatment may be due to the different mechanisms on AFs of these drugs and different drug sensitivity for plant materials. For example, latrunculin $\mathrm{B}$ binds to actin monomers and cytochalasin $\mathrm{D}$ binds to the barbed end of AF to inhibit AF polymerization. Nevertheless, these pharmacological experiments support the necessity of AFs in both stomatal opening and closing. The interconversion and configurations of AFs are highly correlated with the movement of stomatal aperture. Based on orientation, bundling and density, AF configurations in guard cells have been classified into three types during stomatal movement: (1) "radial arrays" or "radial bundles": sparse AFs or bundles are distributed radially from stomatal pores in three-dimensional projection images, and actually cortical AFs are arranged in a circular pattern in cross-sections; (2) "random meshwork": AFs are randomly distributed and organized into mesh-like networks with a high density; and (3) "longitudinal arrays": most AFs form long bundles aligned in the longitudinal direction in guard cells (Gao et al., 2008; Higaki et al., 2010; Li et al., 2014; Shimono et al., 2016; Isner et al., 2017). In general, radial array and radial bundle configurations are more likely to be present in open stomata; filaments are reorganized into promiscuous mesh-like arrangements in the transition state; and longitudinal array configurations are dominant in closed stomata (Higaki et al., 2010; Shimono et al., 2016). Consistently, live-cell imaging of AF configurations in stomatal apertures revealed that $\mathrm{AF}$ remodeling during stomatal movement can be summarized as follows: the cortical radial AFs in open stomata first disassemble and are randomly distributed in response to environmental or endogenous signals, followed by reassembly into long bundles that are parallel to the long axis of guard cells, leading to stomatal closure. By observing AF behaviors in guard cells in the early stage of stomatal closure induced by the bacterial flagellin peptide flg 22 at the singlefilament level, Zou et al. observed that the AF-bundling frequency decreased while the severing frequency increased, which contributed to rapid AF disassembly (Zou et al., 2021). Cotreatment with phalloidin inhibits $\mathrm{ABA}$ or darkness-induced stomatal closure, supporting the notion that AF disassembly may be a critical step for initializing stomatal closure (Gao et al., 2008).

AF dynamics is important factor regulating the activity of ion channels and NADPH oxidase at the membrane that participates in stomata movement. A study in cytochalasin D-treated guard cells evaluated the activity of plasma membrane-localized osmosensitive voltage-dependent inward $\mathrm{K}^{+}$channels and $\mathrm{Ca}^{2+}$-permeable channels at the single-channel level and found that the whole-cell current was increased (Hwang et al., 1997; Liu and Luan, 1998; Zhang and Fan, 2009). In contrast, AF stabilizer phalloidin treatment restrained inward whole-cell $\mathrm{Ca}^{2+}$ current (Zhang and Fan, 2009). Latrunculin $\mathrm{B}$ treatment enhances the vacuolar efflux transient induced by $10 \mu \mathrm{M}$ ABA $\left(\mathrm{Ca}^{2+}\right.$ influx rather than internal $\mathrm{Ca}^{2+}$ release at this concentration of $\mathrm{ABA}$ ) but inhibits that induced by $0.1 \mu \mathrm{M} A B A$ (triggering internal $\mathrm{Ca}^{2+}$ release rather than $\mathrm{Ca}^{2+}$ influx), indicating that AFs also regulate vacuolar ion efflux transient (MacRobbie and Kurup, 2007). The depolymerization of $\mathrm{AFs}$ by latrunculin $\mathrm{B}$ also enhances $\mathrm{ABA}$-induced $\mathrm{H}_{2} \mathrm{O}_{2}$ production through increasing the activity of plasma membrane-localized NADPH oxidase RbohD (Li et al., 2014). Whereas it is still poorly understood how the activity of these proteins is influenced by AF turnover, several possible mechanisms could be tested. A tethering model has been proposed in mammalian cells and Drosophila, where the channel is gated by tethering to the cytoskeleton for mechanosensing (Jiang et al., 2021). For examples, AFs tether Piezo channels in mammalian cells and MTs tether NOMPC channels (belonging to the TRP family) in Drosophila for mechanogating (Zhang et al., 2015; Wang et al., 2020). Whether a similar mechanism whereby the cytoskeleton directly controls the activity of ion channels is conserved in guard cells still awaits further investigation. The distribution patterns and dynamic properties are also critical for membrane protein function. A recent study revealed that AFs and MTs participate in the dynamics of the aquaporin AtPIP2;1 at the plasma membrane during flg22induced stomatal closure. Lat $\mathrm{B}$ treatment promotes the aggregation 
of AtPIP2; 1 at the plasma membrane and accelerates water loss in response to flg22 (Cui et al., 2021). This study provides a new point of view on the activity of plasma membrane proteins regulated by the cytoskeleton.

Vacuoles play a critical role in the regulation of turgor pressure in guard cells. Large vacuoles invaginate to form transvascular strands in opened stomata and split into small vacuoles during stomatal closure, which contributes to changes in the volume of guard cells and the excessive storage of membrane materials (Gao et al., 2005; Tanaka et al., 2007; Yang et al., 2021). AF dynamics is also involved in regulating the morphology of vacuoles in guard cells. AFs colocalize with tonoplasts and encircle small vacuoles (Li et al., 2013). Both depolymerizing or stabilizing AFs by pharmacological agents inhibit the fusion of small vacuoles in guard cells during stomatal opening, as in other cell types (Higaki et al., 2006; Li et al., 2013; Scheuring et al., 2016). SCAB1 is a plant-specific actin-binding protein that can bind, stabilize, and cross-link AFs through dimerization (Zhao et al., 2011; Zhang et al., 2012b; Wang et al., 2015). The mutation of $S C A B 1$ affects the morphological remodeling of the vacuole, and an increased number of transvascular strands appear in the guard cells of scab1 mutants (Yang et al., 2021). The ARP2/3 multisubunit complex, containing two actin-related proteins ARP2 and ARP3, and five other actin-related protein complex units (ARPC1-5), is an important nucleation-promoting and branching factor for AFs (Deeks and Hussey, 2005; Yanagisawa et al., 2013). Vacuole fusion is impaired during stomatal opening in $\mathrm{AF}$ nucleator $\operatorname{arp} 2$ and $\operatorname{arp} 3$ mutants owing to abnormal reorganization of AFs (Li et al., 2013). AFs are also involved in vesicle trafficking from Golgi and release to vacuole (Kim et al., 2005; Akkerman et al., 2011) and the disassembly of AFs affects cargo trafficking from the Golgi complex to the vacuole (Kim et al., 2005). AP3M, the medium subunit of the AP3 complex, serves as an AF-severing protein that participates AF reorganization and vacuole morphology. The mutation of $A P 3 M$ alters $\mathrm{AF}$ status in guard cells and abolishes the transportation of Golgi cargoes, such as the sucrose exporter SUC4, to the tonoplast, leading to defects in stomatal closure under drought stress (Zheng et al., 2019).

\section{RESPONSE OF ACTIN-BINDING PROTEINS TO UPSTREAM SIGNALS IN GUARD CELLS}

Actin-binding proteins (ABPs), which modulate AF nucleation, severing, bundling, polymerization, and depolymerization, alter $\mathrm{AF}$ dynamics to markedly respond to environmental changes (Li et al., 2015). Recent studies have demonstrated that several ABPs are involved in the regulation of stomatal movement. Mutations in the ARP2/3 complex subunits arpc4, arpc5, arpc2 (referred to as $h s r 3$ ), $\operatorname{arp2}$ (referred to as wrm), or arp3 (referred to as dis1) cause a similar phenotype: reduced or abolished dark-, ABA-, and $\mathrm{H}_{2} \mathrm{O}_{2}$-induced stomatal closure and retarded light-induced stomatal opening (Jiang et al., 2012; Li et al., 2013, 2014; Isner et al., 2017). arpc4 and arpc5 mutants show sparser but thicker actin bundles in the guard cells of both opened and closed stomata, suggesting that AFs tend to form bundles in the mutants. AF reorganization is also hysteretic during stomatal closure in the mutants (Li et al., 2014). The ABA-induced mesh-like network organization of AFs is suppressed and thus a more radial array of AFs is retained in the arpc2 mutant compared to the wild type (Jiang et al., 2012). Cytochalasin $\mathrm{D}$ can restore the defect of stomatal closure in response to $\mathrm{ABA}$ in the hsr 3 mutant, suggesting that $\mathrm{ABA}$-induced $\mathrm{AF}$ disassembly is disrupted in $h s r 3$ (Jiang et al., 2012). The ARP2/3 complex is in an intrinsically inactive conformation, which could be converted to an active conformation by the WAVE/SCAR (WASP family Verprolin homologous protein/Suppressor of cAMP Repressor) complex (Frank et al., 2004; Deeks and Hussey, 2005; Yanagisawa et al., 2013). A mutation in the PIR1 gene encoding a subunit of the SCAR/WAVE complex results in reduced dark-induced stomatal closure, while a normal response to $\mathrm{ABA}$ or $\mathrm{CaCl}_{2}$ is retained, and the dark-insensitive phenotype can be restored by latrunculin B or cytochalasin D treatment (Isner et al., 2017). These results indicate that the ARP2/3 complex, along with its upstream regulator, the SCAR/WAVE complex, is required for stomatal movement through their roles in modulating $\mathrm{AF}$ disorganization and remodeling. However, it is still unclear how the ARP2/3 complex and the SCAR/WAVE complex contribute to AF disassembly or rearrangement in guard cells.

Actin-Depolymerizing Factor (ADF) family is a conserved class of ABPs that are involved in plant development and stress responses. The Arabidopsis genome encodes $11 \mathrm{ADF}$ genes categorized into four subclasses (Inada, 2017; Nan et al., 2017), among which most members have conserved actin filament depolymerizing functions, while subclass III members have instead evolved filament bundling functions (Nan et al., 2017). ADF4, a member of subclass I, regulates stomatal closure in response to ABA. The adf4 mutant displays lower AF occupancy but thicker bundles in guard cells than the wild type (Zhao et al., 2016). In contrast, the subclass III member ADF5 regulates drought- and ABA-induced stomatal closure via its AF-bundling activity. $\mathrm{ABA}$ and drought directly promote ADF5 expression mediated by ABF/AREB transcription factors in Arabidopsis and Populus (Qian et al., 2019; Yang et al., 2020). The guard cells of the adf5 mutant exhibit fewer and thinner bundles of actin filaments in open stomata and delayed actin filament reorganization during stomatal closure (Qian et al., 2019). The activity of ADF proteins is governed by many factors, including $\mathrm{pH}$, phosphorylation modifications, and phosphoinositide binding (Inada, 2017). The phosphorylation of the conserved sixth serine (Ser-6) of plant ADF1 and ADF4 inhibits their binding to AFs and therefore abolishes the AF-disassembling activity (Porter et al., 2012; Dong and Hong, 2013). Recently, Shi et al. reported that ABA accumulates and inhibits PP2Cs activity through the ABA-PYLs-PP2Cs complex, resulting in the activation of CKL2 in guard cells (Shi et al., 2021). The ABA-activated CKL2 (Casein Kinase 1-Like Protein 2) phosphorylates ADF4 at Ser-6, which contributes to $\mathrm{AF}$ reorganization in $\mathrm{ABA}$ - and drought-induced stomatal closure (Zhao et al., 2016). More AF-severing events can be observed in the ckl2 mutant, and the severing activity of ADF4 is inhibited in the presence of CKL2 in vitro (Zhao et al., 2016). Ser-6, Ser-105, and Ser-106 of ADF4 can be phosphorylated by calcium-dependent protein kinase 3 (CPK3), which is required for the association with AFs, and stomatal immunity and 
pattern-triggered immunity (Lu et al., 2020). Moreover, the activity of ADFs is pH sensitive (Zhang et al., 2001; Nan et al., 2017; Wioland et al., 2019). As stomatal movement is associated with changes in intracellular $\mathrm{pH}$ due to the influx or efflux of proton, it is plausible to speculate that $\mathrm{pH}$ changes may control stomatal movement at least partially by modulating the activity of ADFs.

Villin belongs to a multifunctional villin/gelsolin/fragmin superfamily that exhibits multiple biochemical activities, including AF bundling, $\mathrm{Ca}^{2+}$-dependent $\mathrm{AF}$ severing, and barbed end capping (Huang et al., 2015). A recent study detailed investigated the functions of Villin3 in stomatal immunity. Zou et al. reported that the vln 3 mutant showed reduced AF turnover in guard cells treated with flg22, resulting in failure to close stomata upon bacterial infection (Zou et al., 2021). Flg22-activated MPK3/MPK6 phosphorylates Villin3 at Ser779 to specifically enhance its severing activity. Neither reduced AF bundling nor increased severing is observed in the guard cells of $v \ln 3$ or $m p k 3 / 6$ double mutant plants in the early stage of flg22 treatment compared to wild type. A phosphorylation mimic version of Villin3 can restore AF dynamics and stomatal movement in the $v \ln 3$ and $m p k 3 / 6$ mutants to the WT level, supporting the importance of VLN3 phosphorylation by MPK3/6 in modulating actin remodeling to activate stomatal defense in Arabidopsis (Zou et al., 2021).

Phosphoinositide exhibits an important function in stomatal movement and ABP activity regulation. The light-induced accumulation of PI $(4,5) \mathrm{P} 2$ triggers stomatal opening (Lee et al., 2007). Phosphatidylinositol 4-phosphate (PI4P), a precursor of $\mathrm{PI}(4,5) \mathrm{P} 2$, and phosphatidylinositol 3-phosphate (PI3P) are required for light-induced stomatal opening and ABA-induced stomatal closure and modulate actin dynamics in guard cells (Jung et al., 2002; Choi et al., 2008). The PI3P and PI4P synthesis inhibitors LY294002 and wortmannin inhibit the ABA-induced random orientation of AF arrays in the guard cells of dayflower (Commelina communis) (Choi et al., 2008). Both overexpression and mutation of the AF cross-linking protein SCAB1 have similar effects, including a reduced rate of actin reorganization and a delay of stomatal closure induced by ABA. However, SCAB1 overexpression results in a higher frequency of bundled actin forms compared to the control, while the scab1 mutant shows similar actin filament reorganization to the wild type (Zhao et al., 2011). Recent research has revealed that SCAB1 binds to PI3P through its RXLR-dEER PI3P-binding motifs. PI3P binding inhibits SCAB1 oligomerization, which further impairs AF destabilization and reorganization during ABA-induced stomatal closure (Yang et al., 2021). Several members of other ABP families, such as Villins and ADFs, as well as the upstream regulator of ARP2/3 complex, the WAVE/SCAR complex (Xiang et al., 2007; Zhao et al., 2010; Qin et al., 2021), bind to and are regulated by phospholipids. These interactions pose the possibility that phospholipids may regulate stomatal movement via multiple mechanism.

\section{MICROTUBULE ORGANIZATION CHANGES DURING STOMATAL MOVEMENT}

The function of MTs in stomatal movement has long been debated due to conflicting results from different experiments (Assmann and Baskin, 1998; Fukuda et al., 1998; Marcus et al., 2001). Assmann et al. reported that neither the microtubule-destabilizing drug colchicine nor the stabilization drug paclitaxel had any effect on stomatal opening or closing in epidermal peels of Vicia faba (Assmann and Baskin, 1998). Contrary results were observed by Fukuda et al. and Marcus et al., where they showed that the microtubule-destabilizing drugs propyzamide, oryzalin, and trifluralin inhibited stomatal opening and that paclitaxel treatment suppressed stomatal closing in the same material (Fukuda et al., 1998; Marcus et al., 2001). It is still unclear why these experiments resulted in totally distinct conclusion. Nevertheless, accumulating evidences from recent decades favor the notion that the MT arrays participate in stomatal movement. During light-induced stomatal opening, oryzalin treatment blocks stomatal opening (Eisinger et al., 2012a; Qu et al., 2017), while stabilization of microtubules by paclitaxel accelerates stomatal opening in a dose-dependent manner $(\mathrm{Qu}$ et al., 2017). ABA-, darkness-, and NO-induced stomatal closure was markedly inhibited by cotreatment with paclitaxel, but no significant changes were observed when oryzalin was applied (Zhang et al., 2008; Eisinger et al., 2012a; Qu et al., 2017; Biel et al., 2020). It has also been reported that treatment with oryzalin alone affects stomatal closure (Khanna et al., 2014).

Live-cell imaging revealed that the number and arrangement pattern of MTs in guard cells are correlated with the stomatal aperture. In open stomata, MTs radiate from the ventral side to the dorsal side in a more parallel, straighter and denser fashion relative to AF organization (Eisinger et al., 2012a; Qu et al., 2017; Biel et al., 2020). Following stomatal closing, MT structures decrease in number and become diffused. Some studies have shown that MTs completely depolymerized (Qu et al., 2017; Yu et al., 2020), while other studies have indicated that MTs are still present, but with reduced density, in closed stomata (Khanna et al., 2014; Biel et al., 2020; Yu et al., 2020; Dou et al., 2021). In the latter case, observable MTs tend to have a longitudinal arrangement and become crisscrossed or randomly patterned near the ventral side (Fukuda et al., 1998; Lahav et al., 2004; Zhang et al., 2008; Eisinger et al., 2012a; Biel et al., 2020). By using end-binding protein 1 (EB1) to label the growing plus ends of microtubules, it was observed that there were no significant changes in the number of growing ends or the growth velocity rate of microtubules during stomatal closure. This observation suggested that the reduction of microtubule density during stomatal closure was most likely resulted from microtubule disassembly (Eisinger et al., 2012a,b). Recently, increasing observations indicate that cortical microtubules are sensitive to tensile stress (Hamant et al., 2008, 2019; Gorelova et al., 2021). Based on the observations from atomic force microscopy and finite element method simulations, MT organization is found to be consistent with the tensile pattern of guard cell (Sampathkumar et al., 2014; Gorelova et al., 2021). Thus, changes in microtubule organization may be a consequence of stomatal movement.

The functions of MTs in stomata movement are still poorly understood. MTs play essential roles in determining the arrangement of cellulose microfibrils and other non-cellulosic compounds in the cell walls, which provides mechanical properties for stomatal movement (Oda, 2015; Rui and Anderson, 2016; Yi et al., 2018). MT guides the trajectories of the cellulose synthesis complexes 
(CSCs) for cellulose synthesis at the cell surface (Gutierrez et al., 2009). Colocalization between CSCs and MTs is reduced and CSC motility speed increase during dark-induced stomata closure (Rui and Anderson, 2016). During stomatal movement, cellulose in guard cell walls undergoes reorganization from a more diffuse distribution in opened stomata to extensive bundles in the closed state (Rui and Anderson, 2016). MTs also determine the alignment mode of callose deposition and disassembly of MTs by oryzalin disturbs the pattern of callose deposition in the guard cell (Apostolakos et al., 2009). Thus, MT organization may impact on cell wall organization during stomatal movement and the detail mechanism deserves further investigation.

\section{MICROTUBULE-ASSOCIATED PROTEINS AND UPSTREAM SIGNALING IN REGULATING MICROTUBULE ORGANIZATION IN GUARD CELLS}

Several microtubule-associated proteins (MAPs) and their upstream regulators play vital roles in regulating MT organization and stomatal movement. WDL7, a member of the WAVEDAMPENED2 (WVD2)/WVD2-LIKE family, directly binds to and bundles MTs in vitro. WDL7-overexpressing plants show delayed stomatal closure in response to ABA compared to WT plants. MTs are less sensitive to oryzalin- and ABA-induced MT disruption in WDL7-overexpressing guard cells, indicating that WDL7 serves as a MT stabilizer. Consistently, the $w d l 7$ mutant shows impairment of MT assembly and the stomatal opening response to light (Dou et al., 2021). WDL7 protein stability is regulated by ubiquitination. MREL57 (MICROTUBULE RELATED E3 LIGASE 57) directly targets and ubiquitinates WDL7 for degradation. mrel57 mutant exhibits ABA insensitivity of stomatal closure and microtubule disassembly in guard cells (Dou et al., 2021). Several other ubiquitin E3 ligases, including JUL1 (JAV1-ASSOCIATED UBIQUITIN LIGASE1) and COP1 (CONSTITUTIVE PHOTOMORPHOGENIC 1), also participate in MT remodeling during stomatal movement (Khanna et al., 2014). JUL1 mediates ABA-induced microtubule disorganization and stomatal closure downstream of $\mathrm{H}_{2} \mathrm{O}_{2}$ and calcium. JUL1 binds to polymerized microtubules but not tubulin heterodimers (Yu et al., 2020). Darkness- and ABA-induced stomatal closure and MT disassembly are suppressed in the cop 1 mutant, and oryzalin is able to reduce this effect, indicating that the function of COP1 is critical for MT destabilization upon darkness and ABA treatment in guard cells (Mao et al., 2005; Khanna et al., 2014; Chen et al., 2021). It has been reported that COP1 directly ubiquitinates the MT stabilizer WDL3 in hypocotyl cells grown under darkness (Lian et al., 2017). Whether an analogous mechanism exists in guard cells requires further evaluation.

Phosphatidic acid (PA) has emerged as a vital signaling molecule involved in regulating cytoskeletal organization and dynamics under abiotic and biotic stresses, including the regulation of stomatal movement (Pleskot et al., 2013). PA is produced through the hydrolysis of phosphatidylcholine (PC) by phospholipase $\mathrm{D}$ (PLD) or the phosphorylation of diacylglycerol (DAG) by DAG kinase (Testerink and Munnik, 2011; Pleskot et al., 2013). Heat shock triggers ROS production to stimulate the activity of plasma membrane-localized PLD $\delta$, and PLD $\delta$ directly binds to and disassembles MTs and causes stomatal closure under heat stress (Zhang et al., 2017; Song et al., 2020). The mutation of PLD $\alpha 1$ maintains stomatal opening and well-organized MTs in the presence of ABA. Exogenous application of PA but not PC, PE or PS promotes microtubule depolymerization in stomatal cells, suggesting that PA may regulate stomatal movement through its impact on MTs (Jiang et al., 2014). However, the molecular mechanism underlying how PA induces MT depolymerization remains unclear. It has been reported that PA binds to MAP65-1 and promotes MT polymerization and bundling under salt stress (Zhang et al., 2012a). It still needs to be explored whether PA activates a MAP or a signaling pathway to disassemble MTs in guard cells.

SINE1 and SINE2 (SUN-INTERACTING NUCLEAR ENVELOPE PROTEIN 1 AND2), which are two components of the plant LINC (LINKER OF NUCLEOSKELETON AND CYTOSKELETON) complex, are involved in regulating the reorganization of MTs in guard cells during stomatal movement. The loss of function of either SINE1 or SINE2 results in a disordered MT organization in open stomata (Biel et al., 2020). There are fewer MT filaments or bundles in sine 1 or sine 2 mutants compared to the wild type during stomatal closure, leading to insensitivity to ABA-induced stomatal closure (Biel et al., 2020). Translationally controlled tumor protein (TCTP) is a calcium- and tubulin-binding protein, and the binding of calcium facilitates TCTP binding to microtubules. The overexpression of TCTP increases ABA- and calcium-induced stomatal closure ratios to limit water evaporation by accelerating MT depolymerization (Kim et al., 2012). The detailed characterization of the biochemical activities of SINE1/2 and TCTP toward MTs should be further defined.

\section{CONCLUSION AND PERSPECTIVE}

Based on the knowledge available, we propose a model of current progress about AF and MT dynamics that are regulated by different functional ABPs and MAPs during stomatal movement. In opened stomata, the ARP $2 / 3$ complex is activated by WAVE/ SCAR complex and promotes AF nucleation and branching, contributing to AF network formation. In the transition stage of stomatal closure, AF-severing factors, such ADF4 and Villin3, lead to AF depolymerization, and an increasing content of PI3P inhibits the cross-linking function of SCAB1, promoting the disassembly of the AF network. Subsequently, the phosphorylation of ADF4 inhibits its severing activity, and the upregulation of $A D F 5$ and SCAB1 dimer contributes to the formation of long AF bundles in closed stomata. WDL7 binds and stabiles MT in opened stomata. During ABA-induced stomatal closing, E3 ligase MREL57 ubiquitinates WDL7 for degradation; ABA-triggered calcium influx activated TCTP to stimulate MT disassembly. PLD $\delta$ activated in an $\mathrm{H}_{2} \mathrm{O}_{2}$ - and calcium-dependent manner and disassembles MTs upon heat stress (Figure 1). 


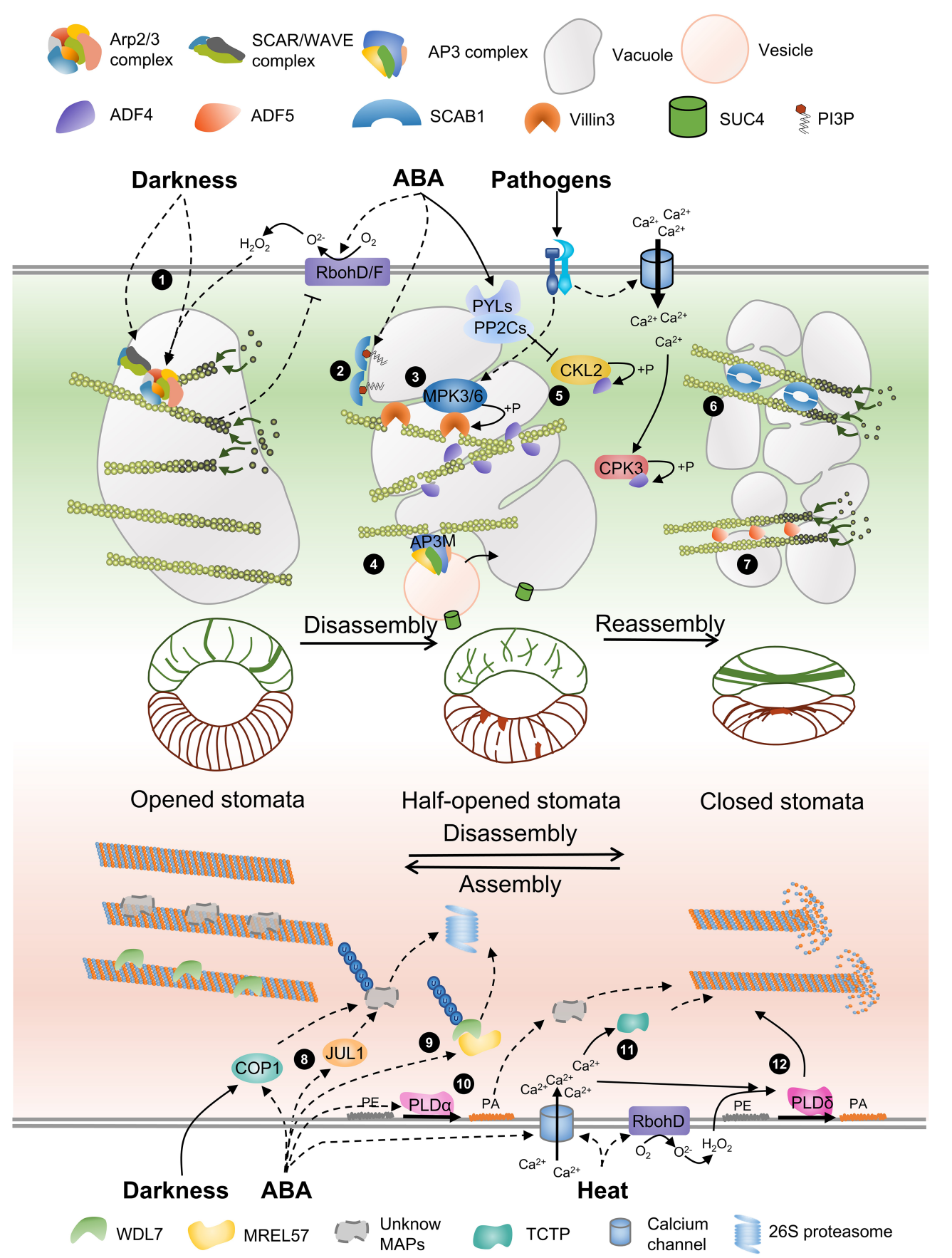

FIGURE 1 | A schematic model of AF and MT remodeling accompanied by vacuole morphology during stomatal closure. The schematic model of stomata in the middle of the panel shows the distribution of AFs and MTs in guard cells with different stomatal apertures. The gray circular charts present the different morphology of vacuole during stomata movement. Several actin-binding proteins and microtubule-associated proteins are involved in regulating cytoskeletal rearrangement. (1) The Arp2/3 complex and the activator SCAR/WAVE complex are required for AF nucleation and branching, and darkness-induced stomata closure. ABA-triggered $\mathrm{H}_{2} \mathrm{O}_{2}$ generation by RbohD/RbohF regulates AF dynamics through the Arp2/3 complex but does not regulate the SCAR/WAVE complex, and AF feedback regulates $\mathrm{H}_{2} \mathrm{O}_{2}$ production. (2) ABA-triggered PI3P biosynthesis inhibits the oligomerization of SCAB1. (3) A pathogen triggers MPK3/MPK6 activation, and Villin3 is then phosphorylated to increase AF-severing activity. (4) AP3M of the AP3 complex severs AFs and regulates vesicles from Golgi carrying SUC4 fused to the tonoplast. (5) ADF4 binds to and severs AFs, and ABA-activated CKL2 and calcium-activated CPK32 induced by pathogens phosphorylate ADF4 to inhibit its activity and promote AF reorganization. (6 and 7) SCAB1 dimers and ADF5 monomers bundle and stabilize AFs and promote AF reassembly. (8) JUL1 and COP1, two other E3 ubiquitin ligases, may control the degradation of unknown MT-stabilizing factors and promote MT disassembly. (9) WDL7 stabilizes MTs in open stomata. The E3 ubiquitin ligase MREL57 interacts with and ubiquitinates WDL7 for 26S proteasome degradation during ABA-induced stomatal closure. (10) ABA-triggered PA produced by PLDa induces MT depolymerization through an unknown mechanism. (11) An ABA-induced increase in cytosolic calcium increases TCTP binding to MTs and MT destabilization. (12) Heat shock stimulates $\mathrm{H}_{2} \mathrm{O}_{2}$ production, and calcium influx activates PLD 8 , which is required for MT depolymerization. 
Although the dynamic distribution of AFs and MTs during stomatal movement has been reported, the underlying molecular mechanism is still not well understood. Additional ABPs, MAPs and upstream proteins involved in stomatal movement need to be detailed analyzed, which will facilitate to dissect the roles of cytoskeleton in transducing environmental signals to stomatal movement. The arrangement mode of AFs and MTs is somewhat similar in guard cells, posing the possibility that they may coordinate to control stomata movement. Some ABPs, such as formin proteins, have been reported to interact with both AFs and MTs (Li et al., 2010; Wang et al., 2013; Sun et al., 2017). It is worthy to further investigate the interaction between AFs and MTs, as well as the underlying molecular mechanism in stomata. Although many evidences have demonstrated the potential for cytoskeleton in regulating the activity of ion channels, vesicles trafficking, and cell wall dynamics of guard cell, the detailed mechanisms need to be explored in further for advancing our understanding of the cytoskeleton function contributed to stomatal movement.

Stomata are of great importance in the response and adaptation of plants to environmental changes. Previous research in this context has mainly focused on kidney-shaped guard cells in

\section{REFERENCES}

Akkerman, M., Overdijk, E. J., Schel, J. H., Emons, A. M., and Ketelaar, T. (2011). Golgi body motility in the plant cell cortex correlates with actin cytoskeleton organization. Plant Cell Physiol. 52, 1844-1855. doi: 10.1093/pcp/pcr122

Apostolakos, P., Livanos, P., and Galatis, B. (2009). Microtubule involvement in the deposition of radial fibrillar callose arrays in stomata of the fern Asplenium nidus L. Cell Motil Cytoskel. 66, 342-349. doi: 10.1002/cm.20366

Assmann, S. M., and Baskin, T. I. (1998). The function of guard cells does not require an intact array of cortical microtubules. J. Exp. Bot. 49, 163-170. doi: $10.1093 / \mathrm{jxb} / 49.319 .163$

Biel, A., Moser, M., and Meier, I. (2020). Arabidopsis KASH proteins SINE1 and SINE2 are involved in microtubule reorganization during ABA-induced stomatal closure. Front. Plant Sci. 11:575573. doi: 10.3389/fpls.2020.575573

Chen, Q., Bai, L., Wang, W., Shi, H., Ramon Botella, J., Zhan, Q., et al. (2021). COP1 promotes ABA-induced stomatal closure by modulating the abundance of ABI/HAB and AHG3 phosphatases. New Phytol. 229, 2035-2049. doi: 10.1111/nph.17001

Choi, Y., Lee, Y., Jeon, B. W., Staiger, C. J., and Lee, Y. (2008). Phosphatidylinositol 3- and 4-phosphate modulate actin filament reorganization in guard cells of day flower. Plant Cell Environ. 31, 366-377. doi: 10.1111/j.1365-3040.2007.01769.x

Cui, Y., Zhao, Y., Lu, Y., Su, X., Chen, Y., Shen, Y., et al. (2021). In vivo singleparticle tracking of the aquaporin AtPIP2;1 in stomata reveals cell type-specific dynamics. Plant Physiol. 185, 1666-1681. doi: 10.1093/plphys/kiab007

Deeks, M. J., and Hussey, P. J. (2005). ARP2/3 and SCAR: plants move to the fore. Nat. Rev. Mol. Cell Biol. 6, 954-964. doi: 10.1038/nrm1765

Dong, C. H., and Hong, Y. (2013). Arabidopsis CDPK6 phosphorylates ADF1 at N-terminal serine 6 predominantly. Plant Cell Rep. 32, 1715-1728. doi: 10.1007/s00299-013-1482-6

Dou, L., He, K., Peng, J., Wang, X., and Mao, T. (2021). The E3 ligase MREL57 modulates microtubule stability and stomatal closure in response to ABA. Nat. Commun. 12:2181. doi: 10.1038/s41467-021-22455-y

Eisinger, W., Ehrhardt, D., and Briggs, W. (2012a). Microtubules are essential for guard cell function in Vicia and Arabidopsis. Mol. Plant 5, 601-610. doi: $10.1093 / \mathrm{mp} / \mathrm{sss} 002$

Eisinger, W. R., Kirik, V., Lewis, C., Ehrhardt, D. W., and Briggs, W. R. (2012b). Quantitative changes in microtubule distribution correlate with guard cell function in Arabidopsis. Mol. Plant 5, 716-725. doi: 10.1093/mp/sss033

Frank, M., Egile, C., Dyachok, J., Djakovic, S., Nolasco, M., Li, R., et al. (2004). Activation of ARP2/3 complex-dependent actin polymerization by plant
Arabidopsis, Vicia, and tobacco. The distribution of AFs and MTs in dumbbell-shaped guard cells in most crop plants is different from that in kidney-shaped guard cells (Spiegelhalder and Raissig, 2021). Advances in research on cytoskeletal dynamics in dumbbell-shaped guard cells remain relatively stagnant. Studies on the mechanisms of stomatal movement in crops will help to improve the efficiency of carbon assimilation and water use under the trend of global warming.

\section{AUTHOR CONTRIBUTIONS}

$\mathrm{YL}$ and $\mathrm{XZ}$ collected the references and wrote the manuscript. YZ and HR revised the manuscript. All authors have read and approved the final manuscript.

\section{FUNDING}

This work was supported by the National Natural Science Foundation of China (91854206 and 32170335 to HR; 31870174 and 32070194 to $\mathrm{YZ}$ ).

proteins distantly related to SCAR/WAVE. Proc. Natl. Acad. Sci. U. S. A. 101, 16379-16384. doi: 10.1073/pnas.0407392101

Fukuda, M., Hasezawa, S., Asai, N., Nakajima, N., and Kondo, N. (1998). Dynamic organization of microtubules in guard cells of Vicia faba L. with diurnal cycle. Plant Cell Physiol. 39:80. doi: 10.1093/oxfordjournals.pcp.a029293

Galatis, B., and Apostolakos, P. (2004). The role of the cytoskeleton in the morphogenesis and function of stomatal complexes. New Phytol. 161, 613-639. doi: 10.1046/j.1469-8137.2003.00986.x

Gao, X. Q., Chen, J., Wei, P. C., Ren, F., Chen, J., and Wang, X. C. (2008). Array and distribution of actin filaments in guard cells contribute to the determination of stomatal aperture. Plant Cell Rep. 27, 1655-1665. doi: 10.1007/s00299-008-0581-2

Gao, X. Q., Li, C. G., Wei, P. C., Zhang, X. Y., and Wang, X. C. (2005). The dynamic changes of tonoplasts in guard cells are important for stomatal movement in Vicia faba. Plant Physiol. 139, 1207-1216. doi: 10.1104/pp.105.067520

Gorelova, V., Sprakel, J., and Weijers, D. (2021). Plant cell polarity as the nexus of tissue mechanics and morphogenesis. Nat. Plants. 7, 1548-1559. doi: 10.1038/s41477-021-01021-w

Gudesblat, G. E., Torres, P. S., and Vojnov, A. A. (2009). Stomata and pathogens: warfare at the gates. Plant Signal. Behav. 4, 1114-1116. doi: 10.4161/psb.4.12.10062

Gutierrez, R., Lindeboom, J. J., Paredez, A. R., Emons, A. M., and Ehrhardt, D. W. (2009). Arabidopsis cortical microtubules position cellulose synthase delivery to the plasma membrane and interact with cellulose synthase trafficking compartments. Nat. Cell Biol. 11, 797-806. doi: 10.1038/ncb1886

Hamant, O., Heisler, M. G., Jonsson, H., Krupinski, P., Uyttewaal, M., Bokov, P., et al. (2008). Developmental patterning by mechanical signals in Arabidopsis. Science 322, 1650-1655. doi: 10.1126/science.1165594

Hamant, O., Inoue, D., Bouchez, D., Dumais, J., and Mjolsness, E. (2019). Are microtubules tension sensors? Nat. Commun. 10:2360. doi: 10.1038/ s41467-019-10207-y

Hayashi, M., Inoue, S., Takahashi, K., and Kinoshita, T. (2011). Immunohistochemical detection of blue light-induced phosphorylation of the plasma membrane $\mathrm{H}^{+}$-ATPase in stomatal guard cells. Plant Cell Physiol. 52, 1238-1248. doi: 10.1093/pcp/pcr072

Hetherington, A. M., and Woodward, F. I. (2003). The role of stomata in sensing and driving environmental change. Nature 424, 901-908. doi: 10.1038/nature01843

Higaki, T., Kutsuna, N., Okubo, E., Sano, T., and Hasezawa, S. (2006). Actin microfilaments regulate vacuolar structures and dynamics: dual observation of actin microfilaments and vacuolar membrane in living tobacco BY-2 cells. Plant Cell Physiol. 47, 839-852. doi: 10.1093/pcp/pcj056 
Higaki, T., Kutsuna, N., Sano, T., Kondo, N., and Hasezawa, S. (2010). Quantification and cluster analysis of actin cytoskeletal structures in plant cells: role of actin bundling in stomatal movement during diurnal cycles in Arabidopsis guard cells. Plant J. 61, 156-165. doi: 10.1111/j.1365-313x.2009.04032.x

Huang, S., Qu, X., and Zhang, R. (2015). Plant villins: versatile actin regulatory proteins. J. Integr. Plant Biol. 57, 40-49. doi: 10.1111/jipb.12293

Hwang, J. U., Suh, S., Yi, H. J., Kim, J., and Lee, Y. (1997). Actin filaments modulate both stomatal opening and inward $\mathrm{K}^{+}$-channel activities in guard cells of Vicia faba L. Plant Physiol. 115, 335-342. doi: 10.1104/Pp.115.2.335

Inada, N. (2017). Plant actin depolymerizing factor: actin microfilament disassembly and more. J. Plant Res. 130, 227-238. doi: 10.1007/s10265-016-0899-8

Isner, J. C., Xu, Z., Costa, J. M., Monnet, F., Batstone, T., Ou, X., et al. (2017). Actin filament reorganisation controlled by the SCAR/WAVE complex mediates stomatal response to darkness. New Phytol. 215, 1059-1067. doi: 10.1111/ nph. 14655

Jiang, K., Sorefan, K., Deeks, M. J., Bevan, M. W., Hussey, P. J., and Hetherington, A. M. (2012). The ARP2/3 complex mediates guard cell actin reorganization and stomatal movement in Arabidopsis. Plant Cell 24, 2031-2040. doi: $10.1105 /$ tpc. 112.096263

Jiang, Y., Wu, K., Lin, F., Qu, Y., Liu, X., and Zhang, Q. (2014). Phosphatidic acid integrates calcium signaling and microtubule dynamics into regulating ABA-induced stomatal closure in Arabidopsis. Planta 239, 565-575. doi: 10.1007/s00425-013-1999-5

Jiang, Y., Yang, X., Jiang, J., and Xiao, B. (2021). Structural designs and mechanogating mechanisms of the mechanosensitive PIEZO channels. Trends Biochem. Sci. 46, 472-488. doi: 10.1016/j.tibs.2021.01.008

Jung, J.-Y., Kim, Y.-W., Kwak, J. M., Hwang, J.-U., Young, J., Schroeder, J. I., et al. (2002). Phosphatidylinositol 3- and 4-phosphate are required for normal stomatal movements. Plant Cell 14, 2399-2412. doi: 10.1105/tpc.004143

Keenan, T. F., Hollinger, D. Y., Bohrer, G., Dragoni, D., Munger, J. W., Schmid, H. P., et al. (2013). Increase in forest water-use efficiency as atmospheric carbon dioxide concentrations rise. Nature 499, 324-327. doi: 10.1038/nature12291

Khanna, R., Li, J., Tseng, T. S., Schroeder, J. I., Ehrhardt, D. W., and Briggs, W. R. (2014). COP1 jointly modulates cytoskeletal processes and electrophysiological responses required for stomatal closure. Mol. Plant 7, 1441-1454. doi: 10.1093/ $\mathrm{mp} / \mathrm{ssu} 065$

Kim, Y. M., Han, Y. J., Hwang, O. J., Lee, S. S., Shin, A. Y., Kim, S. Y., et al. (2012). Overexpression of Arabidopsis translationally controlled tumor protein gene AtTCTP enhances drought tolerance with rapid ABA-induced stomatal closure. Mol. Cells 33, 617-626. doi: 10.1007/s10059-012-0080-8

Kim, M., Hepler, P. K., Eun, S. O., Ha, K. S., and Lee, Y. (1995). Actin filaments in mature guard cells are radially distributed and involved in stomatal movement. Plant Physiol. 109, 1077-1084. doi: 10.1104/pp.109.3.1077

Kim, H., Park, M., Kim, S. J., and Hwang, I. (2005). Actin filaments play a critical role in vacuolar trafficking at the Golgi complex in plant cells. Plant Cell 17, 888-902. doi: 10.1105/tpc.104.028829

Kinoshita, T., Doi, M., Suetsugu, N., Kagawa, T., Wada, M., and Shimazaki, K. (2001). PHOT1 and PHOT2 mediate blue light regulation of stomatal opening. Nature 414:656. doi: 10.1038/414656a

Kinoshita, T., and Shimazaki, K. (1999). Blue light activates the plasma membrane $\mathrm{H}^{+}$-ATPase by phosphorylation of the C-terminus in stomatal guard cells. EMBO J. 18, 5548-5558. doi: 10.1093/emboj/18.20.5548

Kollist, H., Nuhkat, M., and Roelfsema, M. R. (2014). Closing gaps: linking elements that control stomatal movement. New Phytol. 203, 44-62. doi: $10.1111 / \mathrm{nph} .12832$

Lahav, M., Abu-Abied, M., Belausov, E., Schwartz, A., and Sadot, E. (2004). Microtubules of guard cells are light sensitive. Plant Cell Physiol. 45, 573-582. doi: $10.1093 / \mathrm{pcp} / \mathrm{pch} 067$

Lawson, T., and Matthews, J. (2020). Guard cell metabolism and stomatal function. Annu. Rev. Plant Biol. 71, 273-302. doi: 10.1146/annurevarplant-050718-100251

Lebaudy, A., Pascaud, F., Very, A. A., Alcon, C., Dreyer, I., Thibaud, J. B., et al. (2010). Preferential KAT1-KAT2 heteromerization determines inward $\mathrm{K}^{+}$current properties in Arabidopsis guard cells. J. Biol. Chem. 285, 6265-6274. doi: $10.1074 /$ jbc.M109.068445

Lee, Y., Kim, Y. W., Jeon, B. W., Park, K. Y., Suh, S. J., Seo, J., et al. (2007). Phosphatidylinositol 4,5-bisphosphate is important for stomatal opening. Plant J. 52, 803-816. doi: 10.1111/j.1365-313X.2007.03277.x
Li, J., Blanchoin, L., and Staiger, C. J. (2015). Signaling to actin stochastic dynamics. Annu. Rev. Plant Biol. 66, 415-440. doi: 10.1146/annurevarplant-050213-040327

Li, X., Li, J. H., Wang, W., Chen, N. Z., Ma, T. S., Xi, Y. N., et al. (2014). ARP2/3 complex-mediated actin dynamics is required for hydrogen peroxideinduced stomatal closure in Arabidopsis. Plant Cell Environ. 37, 1548-1560. doi: $10.1111 /$ pce. 12259

Li, L. J., Ren, F., Gao, X. Q., Wei, P. C., and Wang, X. C. (2013). The reorganization of actin filaments is required for vacuolar fusion of guard cells during stomatal opening in Arabidopsis. Plant Cell Environ. 36, 484-497. doi: 10.1111/j.1365-3040.2012.02592.x

Li, Y., Shen, Y., Cai, C., Zhong, C., Zhu, L., Yuan, M., et al. (2010). The type II Arabidopsis formin14 interacts with microtubules and microfilaments to regulate cell division. Plant Cell 22, 2710-2726. doi: 10.1105/tpc.110.075507

Lian, N., Liu, X., Wang, X., Zhou, Y., Li, H., Li, J., et al. (2017). COP1 mediates dark-specific degradation of microtubule-associated protein WDL3 in regulating Arabidopsis hypocotyl elongation. Proc. Natl. Acad. Sci. U. S. A. 114, 12321-12326. doi: 10.1073/pnas.1708087114

Liu, K., and Luan, S. (1998). Voltage-dependent $\mathrm{K}^{+}$channels as targets of osmosensing in guard cells. Plant Cell 10, 1957-1970. doi: 10.1105/tpc.10.11.1957

Lu, Y. J., Li, P., Shimono, M., Corrion, A., Higaki, T., He, S. Y., et al. (2020). Arabidopsis CALCIUM-DEPENDENT PROTEIN KINASE 3 regulates actin cytoskeleton organization and immunity. Nat. Commun. 11:6234. doi: 10.1038/ s41467-020-20007-4

MacRobbie, E. A. C., and Kurup, S. (2007). Signalling mechanisms in the regulation of vacuolar ion release in guard cells. New Phytol. 175, 630-640. doi: $10.1111 / \mathrm{j} .1469-8137.2007 .02131 . \mathrm{x}$

Mao, J., Zhang, Y. C., Sang, Y., Li, Q. H., and Yang, H. Q. (2005). From the cover: a role for Arabidopsis cryptochromes and COP1 in the regulation of stomatal opening. Proc. Natl. Acad. Sci. U. S. A. 102, 12270-12275. doi: 10.1073/pnas.0501011102

Marcus, A. I., Moore, R. C., and Cyr, R. J. (2001). The role of microtubules in guard cell function. Plant Physiol. 125, 387-395. doi: 10.1104/Pp.125.1.387

Melotto, M., Zhang, L., Oblessuc, P. R., and He, S. Y. (2017). Stomatal defense a decade later. Plant Physiol. 174, 561-571. doi: 10.1104/pp.16.01853

Murata, Y., Mori, I. C., and Munemasa, S. (2015). Diverse stomatal signaling and the signal integration mechanism. Annu. Rev. Plant Biol. 66, 369-392. doi: 10.1146/annurev-arplant-043014-114707

Muroyama, A., Gong, Y., and Bergmann, D. C. (2020). Opposing, polaritydriven nuclear migrations underpin asymmetric divisions to pattern Arabidopsis stomata. Curr. Biol. 30, 4467.e4-4475.e4. doi: 10.1016/j.cub.2020.08.100

Nan, Q., Qian, D., Niu, Y., He, Y., Tong, S., Niu, Z., et al. (2017). Plant actindepolymerizing factors possess opposing biochemical properties arising from key amino acid changes throughout evolution. Plant Cell 29, 395-408. doi: $10.1105 /$ tpc. 16.00690

Oda, Y. (2015). Cortical microtubule rearrangements and cell wall patterning. Front. Plant Sci. 6:236. doi: 10.3389/fpls.2015.00236

Panteris, E., Achlati, T., Daras, G., and Rigas, S. (2018). Stomatal complex development and F-actin organization in maize leaf epidermis depend on cellulose synthesis. Molecules 23:1365. doi: 10.3390/molecules23061365

Pleskot, R., Li, J., Zarsky, V., Potocky, M., and Staiger, C. J. (2013). Regulation of cytoskeletal dynamics by phospholipase D and phosphatidic acid. Trends Plant Sci. 18, 496-504. doi: 10.1016/j.tplants.2013.04.005

Porter, K., Shimono, M., Tian, M., and Day, B. (2012). Arabidopsis ACTINDEPOLYMERIZING FACTOR-4 links pathogen perception, defense activation and transcription to cytoskeletal dynamics. PLoS Pathog. 8:e1003006. doi: 10.1371/journal.ppat.1003006

Qian, D., Zhang, Z., He, J., Zhang, P., Ou, X., Li, T., et al. (2019). Arabidopsis ADF5 promotes stomatal closure by regulating actin cytoskeleton remodeling in response to $\mathrm{ABA}$ and drought stress. J. Exp. Bot. 70, 435-446. doi: $10.1093 / \mathrm{jxb} / \mathrm{ery} 385$

Qin, L., Liu, L., Tu, J., Yang, G., Wang, S., Quilichini, T. D., et al. (2021). The ARP2/3 complex, acting cooperatively with class I formins, modulates penetration resistance in Arabidopsis against powdery mildew invasion. Plant Cell 33, 3151-3175. doi: 10.1093/plcell/koab170

Qu, Y., Song, P., Hu, Y., Jin, X., Jia, Q., Zhang, X., et al. (2017). Regulation of stomatal movement by cortical microtubule organization in response to darkness and ABA signaling in Arabidopsis. Plant Growth Regul. 84, 467-479. doi: 10.1007/s10725-017-0353-5 
Rui, Y., and Anderson, C. T. (2016). Functional analysis of cellulose and xyloglucan in the walls of stomatal guard cells of Arabidopsis. Plant Physiol. 170, 1398-1419. doi: 10.1104/pp.15.01066

Sampathkumar, A., Krupinski, P., Wightman, R., Milani, P., Berquand, A., Boudaoud, A., et al. (2014). Subcellular and supracellular mechanical stress prescribes cytoskeleton behavior in Arabidopsis cotyledon pavement cells. Elife 3:e01967. doi: 10.7554/eLife.01967

Scheuring, D., Lofke, C., Kruger, F., Kittelmann, M., Eisa, A., Hughes, L., et al. (2016). Actin-dependent vacuolar occupancy of the cell determines auxininduced growth repression. Proc. Natl. Acad. Sci. U. S. A. 113, 452-457. doi: $10.1073 /$ pnas. 1517445113

Shi, Y., Liu, X., Zhao, S., and Guo, Y. (2021). The PYR-PP2C-CKL2 module regulates ABA-mediated actin reorganization during stomatal closure. New Phytol. 233, 2168-2184. doi: 10.1111/nph.17933

Shimono, M., Higaki, T., Kaku, H., Shibuya, N., Hasezawa, S., and Day, B. (2016). Quantitative evaluation of stomatal cytoskeletal patterns during the activation of immune signaling in Arabidopsis thaliana. PLoS One 11:e0159291. doi: 10.1371/journal.pone.0159291

Song, P., Jia, Q., Chen, L., Jin, X., Xiao, X., Li, L., et al. (2020). Involvement of Arabidopsis phospholipase D $\delta$ in regulation of ROS-mediated microtubule organization and stomatal movement upon heat shock. J. Exp. Bot. 71, 6555-6570. doi: 10.1093/jxb/eraa359

Spiegelhalder, R. P., and Raissig, M. T. (2021). Morphology made for movement: formation of diverse stomatal guard cells. Curr. Opin. Plant Biol. 63:102090. doi: 10.1016/j.pbi.2021.102090

Sun, T., Li, S., and Ren, H. (2017). OsFH15, a class I formin, interacts with microfilaments and microtubules to regulate grain size via affecting cell expansion in rice. Sci. Rep. 7:6538. doi: 10.1038/s41598-017-06431-5

Tanaka, Y., Kutsuna, N., Kanazawa, Y., Kondo, N., Hasezawa, S., and Sano, T. (2007). Intra-vacuolar reserves of membranes during stomatal closure: the possible role of guard cell vacuoles estimated by $3-\mathrm{D}$ reconstruction. Plant Cell Physiol. 48, 1159-1169. doi: 10.1093/pcp/pcm085

Testerink, C., and Munnik, T. (2011). Molecular, cellular, and physiological responses to phosphatidic acid formation in plants. J. Exp. Bot. 62, 2349-2361. doi: $10.1093 /$ jxb/err079

Wang, J., Jiang, J., Yang, X., Wang, L., and Xiao, B. (2020). Tethering Piezo channels to the actin cytoskeleton for mechanogating via the E-cadherin$\beta$-catenin mechanotransduction complex. bioRxiv [Preprint].

Wang, J., Zhang, Y., Wu, J., Meng, L., and Ren, H. (2013). AtFH16, an Arabidopsis type II formin, binds and bundles both microfilaments and microtubules, and preferentially binds to microtubules. J. Integr. Plant Biol. 55, 1002-1015. doi: $10.1111 /$ jipb.12089

Wang, C., Zheng, Y., Zhao, Y., Zhao, Y., Li, J., and Guo, Y. (2015). SCAB3 is required for reorganization of actin filaments during light quality changes. J. Genet. Genomics 42, 161-168. doi: 10.1016/j.jgg.2015.02.005

Wioland, H., Jegou, A., and Romet-Lemonne, G. (2019). Quantitative variations with ph of actin depolymerizing factor/cofilin's multiple actions on actin filaments. Biochemistry 58, 40-47. doi: 10.1021/acs.biochem.8b01001

Woolfenden, H. C., Baillie, A. L., Gray, J. E., Hobbs, J. K., Morris, R. J., and Fleming, A. J. (2018). Models and mechanisms of stomatal mechanics. Trends Plant Sci. 23, 822-832. doi: 10.1016/j.tplants.2018.06.003

Xiang, Y., Huang, X., Wang, T., Zhang, Y., Liu, Q., Hussey, P. J., et al. (2007). ACTIN BINDING PROTEIN 29 from Lilium pollen plays an important role in dynamic actin remodeling. Plant Cell 19, 1930-1946. doi: 10.1105/tpc.106.048413

Yamauchi, S., Takemiya, A., Sakamoto, T., Kurata, T., Tsutsumi, T., Kinoshita, T., et al. (2016). The plasma membrane $\mathrm{H}^{+}$-ATPase AHAl plays a major role in stomatal opening in response to blue light. Plant Physiol. 171, 2731-2743. doi: $10.1104 / p p \cdot 16.01581$

Yanagisawa, M., Zhang, C., and Szymanski, D. B. (2013). ARP2/3-dependent growth in the plant kingdom: SCARs for life. Front. Plant Sci. 4:166. doi: $10.3389 /$ fpls.2013.00166

Yang, Y., Li, H. G., Wang, J., Wang, H. L., He, F., Su, Y., et al. (2020). ABF3 enhances drought tolerance via promoting ABA-induced stomatal closure by directly regulating ADF5 in Populus euphratica. J. Exp. Bot. 71, 7270-7285. doi: $10.1093 / \mathrm{jxb} / \mathrm{eraa} 383$

Yang, Y., Yang, Y., Zhao, Y., Zheng, W., Zhao, Y., Zhao, S., et al. (2021). Phosphatidylinositol 3-phosphate regulates SCAB1-mediated F-actin reorganization during stomatal closure in Arabidopsis. Plant Cell 34, 477-494. doi: $10.1093 /$ plcell/koab264
Yi, H., Rui, Y., Kandemir, B., Wang, J. Z., Anderson, C. T., and Puri, V. M. (2018). Mechanical effects of cellulose, xyloglucan, and pectins on stomatal guard cells of Arabidopsis thaliana. Front. Plant Sci. 9:1566. doi: 10.3389/fpls.2018.01566

Yu, S. G., Kim, J. H., Cho, N. H., Oh, T. R., and Kim, W. T. (2020). Arabidopsis RING E3 ubiquitin ligase JUL1 participates in ABA-mediated microtubule depolymerization, stomatal closure, and tolerance response to drought stress. Plant J. 103, 824-842. doi: 10.1111/tpj.14775

Zeng, W., Melotto, M., and He, S. Y. (2010). Plant stomata: a checkpoint of host immunity and pathogen virulence. Curr. Opin. Biotechnol. 21, 599-603. doi: 10.1016/j.copbio.2010.05.006

Zhang, X., Dong, F. C., Gao, J. F., and Song, C. P. (2001). Hydrogen peroxideinduced changes in intracellular $\mathrm{pH}$ of guard cells precede stomatal closure. Cell Res. 11, 37-43. doi: 10.1038/sj.cr.7290064

Zhang, W., and Fan, L. M. (2009). Actin dynamics regulates voltage-dependent calcium-permeable channels of the Vicia faba guard cell plasma membrane. J. Integr. Plant Biol. 51, 912-921. doi: 10.1111/j.1744-7909.2009.00859.x

Zhang, Q., Lin, F., Mao, T., Nie, J., Yan, M., Yuan, M., et al. (2012a). Phosphatidic acid regulates microtubule organization by interacting with MAP65-1 in response to salt stress in Arabidopsis. Plant Cell 24, 4555-4576. doi: 10.1105/ tpc.112.104182

Zhang, Q., Song, P., Qu, Y., Wang, P., Jia, Q., Guo, L., et al. (2017). Phospholipase D $\delta$ negatively regulates plant thermotolerance by destabilizing cortical microtubules in Arabidopsis. Plant Cell Environ. 40, 2220-2235. doi: 10.1111/pce.13023

Zhang, W., Cheng, L. E., Kittelmann, M., Li, J., Petkovic, M., Cheng, T., et al. (2015). Ankyrin repeats convey force to gate the NOMPC mechanotransduction channel. Cell 162, 1391-1403.

Zhang, Y., Wu, Z., Wang, X., and Yu, R. (2008). Rearrangements of microtubule cytoskeleton in stomatal closure of Arabidopsis induced by nitric oxide. Sci. Bull. 53, 848-852. doi: 10.1007/s11434-008-0142-7

Zhang, W., Zhao, Y., Guo, Y., and Ye, K. (2012b). Plant actin-binding protein SCAB1 is dimeric actin cross-linker with a typical pleckstrin homology domain. J. Biol. Chem. 287, 11981-11990. doi: 10.1074/jbc.M111.338525

Zhao, H., Hakala, M., and Lappalainen, P. (2010). ADF/cofilin binds phosphoinositides in a multivalent manner to act as a $\mathrm{PIP}_{2}$-density sensor. Biophys. J. 98, 2327-2336. doi: 10.1016/j.bpj.2010.01.046

Zhao, S., Jiang, Y., Zhao, Y., Huang, S., Yuan, M., Zhao, Y., et al. (2016) CASEIN KINASE1-LIKE PROTEIN2 regulates actin filament stability and stomatal closure via phosphorylation of actin depolymerizing factor. Plant Cell 28, 1422-1439. doi: 10.1105/tpc.16.00078

Zhao, Y., Zhao, S., Mao, T., Qu, X., Cao, W., Zhang, L., et al. (2011). The plant-specific actin binding protein SCAB1 stabilizes actin filaments and regulates stomatal movement in Arabidopsis. Plant Cell 23, 2314-2330. doi: $10.1105 /$ tpc. 111.086546

Zheng, W., Jiang, Y., Wang, X., Huang, S., Yuan, M., and Guo, Y. (2019) AP3M harbors actin filament binding activity that is crucial for vacuole morphology and stomatal closure in Arabidopsis. Proc. Natl. Acad. Sci. U. S. A. 116, 18132-18141. doi: 10.1073/pnas.1901431116

Zou, M., Guo, M., Zhou, Z., Wang, B., Pan, Q., Li, J., et al. (2021). MPK3and MPK6-mediated VLN3 phosphorylation regulates actin dynamics during stomatal immunity in Arabidopsis. Nat. Commun. 12:6474. doi: 10.1038/ s41467-021-26827-2

Conflict of Interest: The authors declare that the research was conducted in the absence of any commercial or financial relationships that could be construed as a potential conflict of interest.

Publisher's Note: All claims expressed in this article are solely those of the authors and do not necessarily represent those of their affiliated organizations, or those of the publisher, the editors and the reviewers. Any product that may be evaluated in this article, or claim that may be made by its manufacturer, is not guaranteed or endorsed by the publisher.

Copyright (c) $2022 \mathrm{Li}$, Zhang, Zhang and Ren. This is an open-access article distributed under the terms of the Creative Commons Attribution License (CC BY). The use, distribution or reproduction in other forums is permitted, provided the original author(s) and the copyright owner(s) are credited and that the original publication in this journal is cited, in accordance with accepted academic practice. No use, distribution or reproduction is permitted which does not comply with these terms. 